\title{
Pharmacogenetics-based warfarin dosing in children
}

Clinical factors, demographic variables and variations in two genes, CYP2C9 and VKORC1, have been shown to contribute to the variability in warfarin dose requirements among adult patients. Less is known about their relative importance for dose variability in children. A few small studies have been reported, but the results have been conflicting, especially regarding the impact of genotypes. In this article, we critically review published pharmacogenetic-based prediction models for warfarin dosing in children, and present results from a head-to-head comparison of predictive performance in a distinct cohort of warfarin-treated children. Finally we discuss what properties a prediction model should have, and what knowledge gaps need to be filled, to improve warfarin therapy in children of all ages.

\section{KEYWORDS: children dose individualization pharmacogenetics PK/PD models prediction models warfarin}

Warfarin is the most widely prescribed anticoagulant for the prevention and treatment of thromboembolic events in both adults [1] and children [2]. Although effective, the use of warfarin is limited by a narrow therapeutic range combined with a large difference in the dose required for adequate anticoagulation. To achieve a favorable balance between the desired antithrombotic effect and the risk of bleeding, it is necessary to identify the right dose for each patient. The challenge is to predict the right dose beforehand, or to correctly interpret early measurements of the international normalized ratio (INR) following a standard dose and to adjust the dose to maintain INR within the therapeutic range. Target INR ranges for children are extrapolated from studies conducted in adults [2]. For most indications, the target INR range is 2.0-3.0, but there are also low target indications (range: 1.5-1.9) and high target indications, for example, prosthetic heart valve patients with a target INR from 2.5 to 4.0 [2]. Variability in both pharmacokinetics (PK) and pharmacodynamics (PD) has been shown to contribute to the more than 20 -fold difference in therapeutic dose in adults [3]. Part of this variability has been ascribed to clinical and demographic factors, such as age, body size, comorbidities, comedications and dietary vitamin $\mathrm{K}$ intake [1]. There is extensive evidence to support that up to $40 \%$ of the dose variability in adults is explained by SNPs in the two genes CYP2C9 ( ${ }^{*} 2$ and $\left.{ }^{*} 3\right)$ and VKORC1 $(-1639 \mathrm{G}>\mathrm{A}$ or $1173 \mathrm{C}>\mathrm{T})[4-8]$. These findings have resulted in several updates of the US FDA-approved warfarin label, and from
2010 include a recommendation that warfarin dosing could be guided by the patient's $C Y P 2 C 9$ and VKORC1 genotype if this is known [101]. The potential clinical importance of a patient's genotype has been described in retrospective observational studies in both pediatric [9] and adult patients [8,10-13], where carriers of at least one variant allele were found to be predisposed to unstable anticoagulation and an increased risk of bleeding, especially during the initiation of therapy.

The aims of this review are to describe the two main types of warfarin dose prediction models and some of their respective advantages and limitations, to review published pharmacogenetic-based prediction models for warfarin dosing in children, to provide results from a head-to-head comparison of pharmacogeneticbased prediction models for warfarin dosing in children, to discuss important properties of a prediction model for warfarin dosing in children, and to identify knowledge gaps that need to be filled to improve warfarin therapy in children of all ages. Although an extensive literature search was made using electronic databases, only references considered to illustrate specific concepts or findings are quoted.

\section{Warfarin dose prediction models} Different types of prediction models Linear regression models

The vast majority of published pharmacogenetic prediction models for warfarin are linear regression models [6-8,14-17]. These models are based on multiple linear regressions that associate

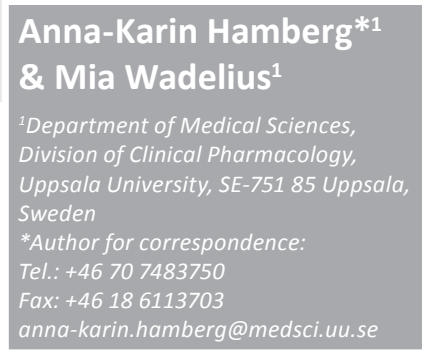

Future
Medicine $\mathrm{fs}$ par of 
interindividual variability in stable warfarin dose with genetic and/or clinical characteristics. Advantages of linear regression models include that they are standard methods in the field of medicine, and the necessary competence is readily available; model development is relatively quick and straight forward; only a limited amount of data is required, with dose information from a single time point per patient collected at steady state being sufficient; and the output from the analysis represents a closed form solution that can be converted to a simple equation that is easy to use. However, the empirical and descriptive nature of these models results in some limitations. First, the models fail to explain the causal relationship between the variability in dose and the predictors. Second, by limiting observations to steady state, the models will only be able to address variability in extent, and not rate, of response. Third, the empirical nature of a linear regression model limits its use to the patient population on which it was developed $[18,19]$. Furthermore, large sample sizes are required to get accurate point estimates of the effect of predictors that are less common in the population, for example, the effect of rare genotypes. A further limitation is that most regression models are limited to the prediction of maintenance doses. As previously described by Eriksson and Wadelius [18], individualization of warfarin therapy should also include the prediction of initiation doses [20] and dose revisions [21] that incorporate information from previous doses and INR measurements into the prediction model.

\section{PK/PD-based models}

Population PK/PD modeling, or pharmacometrics, has emerged as an important tool for quantitating and explaining variability in drug exposure and response. Pharmacometrics uses mathematical and statistical models to characterize, understand, and predict a drug's pharmacokinetic, pharmacodynamic and biomarkeroutcome behavior on a population level, but also for individual patients [22]. Dose-prediction models for warfarin based on PK/PD principles and including pharmacogenetic predictors of variability have been developed on data from large studies in adults by Hamberg et al. [3,23], Lindner et al. [24] and Perlstein et al. [25]. These models are mechanistically based, and therefore have advantages over the descriptive regression models. First, the models can estimate both temporal and quantitative aspects of drug response, and thereby address variability in both the rate and extent of the response. This makes it possible to also account for the time delay between PK and PD that for warfarin is quite pronounced $[3,26]$. Second, the models are not limited to the patient population on which they were developed. Knowledge gained in one population can be extrapolated to another population through the use of pharmacological principles, for example, from adults to children. Third, the same model can be used for prediction of both an initiation dose and an expected maintenance dose, so called a priori predictions, and for dose revisions, so called a posteriori predictions. Other advantages of the PK/PD models are the abilities to provide dose predictions for any target INR, to adjust for differences in baseline INR and to handle dose predictions under nonsteady-state conditions [27]. However, there are limitations associated with this type of model. First, the preparation of datasets and model development can be time consuming and requires a highly specialized competence. Second, the treatment data included in the analysis should cover all phases of therapy to accurately capture variability in both the rate and extent of response. Third, the models often require software to solve a set of differential equations without a closed form solution in order to provide individualized dose predictions.

Figure 1 highlights the main differences between an empirical linear regression model and a mechanistically based PK/PD model, and how the two models incorporate the effect of CYP2C9 and VKORC1 genotypes in the dose response to warfarin.

\section{Prediction models in children}

The simplest prediction model for warfarin dosing in children is a weight-based dose model, with a starting dose of $0.2 \mathrm{mg} / \mathrm{kg} /$ day and subsequent dose adjustments that are based on the individual INR response [2].

\section{Linear regression models}

Several recent studies have evaluated the influence of pharmacogenetics and other factors on warfarin dose variability in children [14-17,28]. The number of children included in individual studies has ranged from 34 to 120 . Details about demographics and genotypes for children included in the studies are summarized in Table 1. Each study developed a linear regression model for the prediction of maintenance dose in children. The individual prediction and regression models are summarized in TABLE 2. Interesting observations are that all models except one [28] include predictions for all three possible $V K O R C 1$ genotypes, and that only the model by 
Biss et al. includes predictions for all six investigated CYP2C9 genotypes [17]. The model developed on data from Japanese children [28] did not include CYP2C9 genotype effects since only one of the 48 children carried a variant allele. One model only differentiated between wildtype and nonwild-type genotypes [14], another discriminated between subjects carrying ${ }^{*} 1 /{ }^{*} 1$, ${ }^{*} 1 /{ }^{*} 2$ and other genotypes $\left({ }^{*} 1 /{ }^{*} 3,{ }^{*} 2 /{ }^{*} 2,{ }^{*} 2 /{ }^{*} 3\right.$ or $* 3 / * 3$ ) [16], while a third model divided subjects into wild-type, one variant allele and two variant alleles, but made no distinction between the effect of the *2 or *3 allele [15]. These distinctions were probably owing to the limited sample sizes, with very few children having ${ }^{*} 2$ and/or *3 variant alleles. Looking across all studies the total number of children with $C Y P 2 C 9^{*} 2 /{ }^{*} 2$, ${ }^{*} 2 /{ }^{*} 3$ or ${ }^{*} 3 /{ }^{*} 3$ genotype were only 5,2 and 1 , respectively (see TABLE 1).

\section{PK/PD-based models}

Population PK/PD models can alleviate many of the difficulties encountered when analyzing data from pediatric studies, and can help transform variability in PK and PD into individualized dosing recommendations. The possibility to bridge from an existing adult PK/PD model to pediatrics based on pharmacological principles has been applied to warfarin by both Hamberg et al. [27] and Lala et al. [29]. In both cases, adult PK/PD models [23,102] were used to derive pediatric PK/PD models by taking into account the impact of body size on drug clearance (CL) and volume of distribution (V) [30]; the maturation pattern of the metabolizing enzymes involved in warfarin disposition [31]; and the mechanism of action of warfarin. Both models have been evaluated in a pediatric dataset, but note that no data from warfarin-treated children were used for the derivation of these models [27,29].

Individual predictors in the Hamberg model included CYP2C9 and VKORC1 genotype, age (to account for maturation of the CYP2C9 enzyme), bodyweight (in a nonlinear relation to $C L$ and in a linear relation to $V$ ), target INR and baseline INR (seeing the effect of warfarin as a change from baseline) [27]. Predictors in the Lala model included CYP2C9 and VKORC1 genotype, age (to account for maturation of the CYP2C9 enzyme) and bodyweight (in a nonlinear relation to $\mathrm{CL}$ and in a linear relation to $\mathrm{V})[29]$.

\section{Evaluation of prediction models}

Model evaluation can be classified as internal and external. Internal evaluation means that
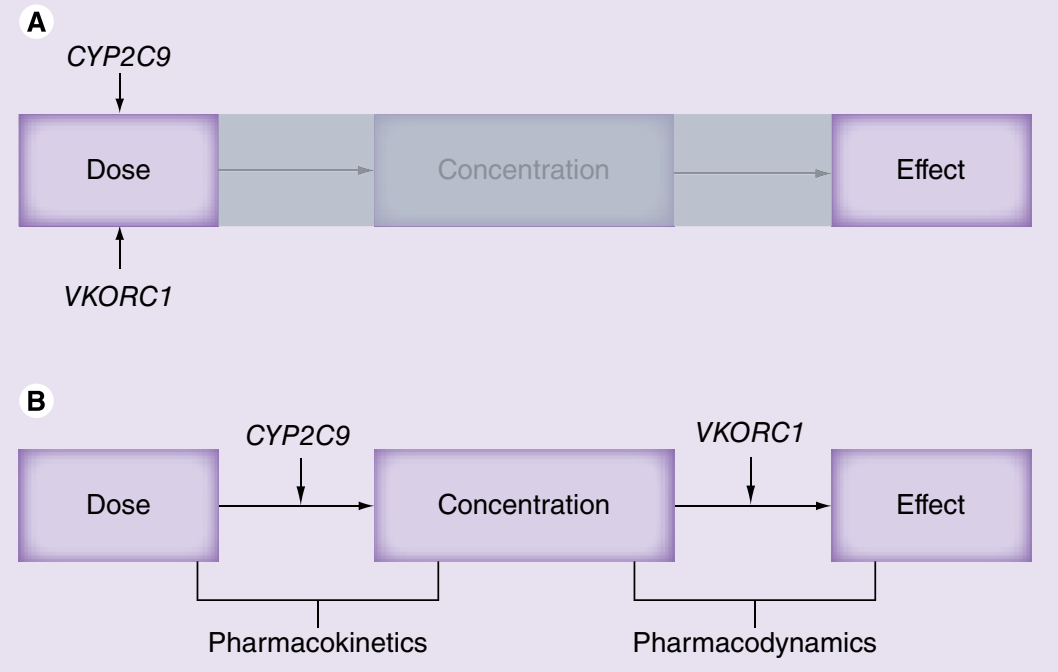

Figure 1. How the effect of CYP2C9 and VKORC1 genotype on warfarin dose requirement differs in different models. (A) A descriptive linear regression model where concentration is ignored and both CYP2C9 and VKORC1 genotype effects are placed on dose. (B) A mechanism-based pharmacokinetic/pharmacodynamic model.

the same data is used for model evaluation, for example, by using simulation-based techniques, while an external evaluation is performed on data from a new and independent study. A common problem with external evaluation is the difficulty to find a second dataset that is similar in design (e.g., target population, definition of treatment target) and performance (e.g., data quality, analytical methods) as the original dataset. An alternative approach is to randomly split a dataset into two parts; one for model building and one for model evaluation. However, there are some drawbacks with this approach, especially when the study population is small and heterogeneous, which is often the case for studies conducted in children. Since only part of the data is used for model development, the parameter estimates will be less informed and thereby less precise than when using all data available. Results from a simulation study have indicated that internal model evaluation is preferred over data splitting, with the exception of very small studies $(\mathrm{n}<25)$ [103].

\section{Common performance measures Linear regression models}

The most common performance measure of linear regression models is the coefficient of determination $\left(\mathrm{R}^{2}\right)$. $\mathrm{R}^{2}$ measures the percentage (or proportion) of variability of the outcome that is explained by individual predictors (univariate analysis) or by the full model (multivariate analysis). While the $\mathrm{R}^{2}$-value gives a point estimate for the predictive ability of the 


\section{Table 1. Demographics of children included in the development of five linear regression models and a}

validation dataset.

\begin{tabular}{|c|c|c|c|c|c|c|}
\hline & $\begin{array}{l}\text { Nowak-Göttl } \\
\text { et al. }[14] \\
\mathbf{n}=\mathbf{5 9}(34 \text { on } \\
\text { warfarin) }\end{array}$ & $\begin{array}{l}\text { Moreau et al. } \\
{[15], \mathbf{n}=\mathbf{8 3}}\end{array}$ & $\begin{array}{l}\text { Biss et al. } \\
\mathbf{n}=120\end{array}$ & $\begin{array}{l}\text { Nguyen et al. } \\
{[16], \mathbf{n}=\mathbf{3 7}}\end{array}$ & $\begin{array}{l}\text { Kato et al. } \\
{[28], \mathbf{n}=\mathbf{4 8}}\end{array}$ & $\begin{array}{l}\text { Validation } \\
\text { dataset [27], } \\
\mathbf{n}=31^{\dagger}\end{array}$ \\
\hline Sex, male/female (n) & $27 / 32$ & $46 / 37$ & $82 / 38$ & $26 / 11$ & $33 / 15$ & $18 / 13$ \\
\hline Median age, years (range) & $\begin{array}{l}15.0 \\
(1.0-19.0)\end{array}$ & $\begin{array}{l}8.4^{\ddagger} \\
(3 \text { months }-18)\end{array}$ & $\begin{array}{l}11.7 \\
(1.75-18.6)\end{array}$ & $\begin{array}{l}9.6^{\ddagger} \\
(1.8-18.6)\end{array}$ & $\begin{array}{l}6.6^{\ddagger} \\
(0.42-19.2)\end{array}$ & $\begin{array}{l}3.2 \\
(0.33-15.6)\end{array}$ \\
\hline Median weight, kg (range) & $\begin{array}{l}61.0 \\
(2.3-101)\end{array}$ & $\begin{array}{l}29.5^{\ddagger} \\
(3.5-81.5)\end{array}$ & $\begin{array}{l}37.0 \\
(11.7-100)\end{array}$ & $\begin{array}{l}37.8^{\ddagger} \\
(7.7-95)\end{array}$ & $19.7^{\ddagger}$ & $\begin{array}{l}14.2 \\
(4.8-60.7)\end{array}$ \\
\hline Median height, cm (range) & No data & $121^{\ddagger}(50-183)$ & $143(79-196)$ & $(66-202)$ & No data & $94(62-180)$ \\
\hline \multicolumn{7}{|l|}{ CYP2C9 genotype ${ }^{\S}, n(\%)$} \\
\hline$* 1 / * 1$ & $39(66)$ & $53(64)$ & $84(70)$ & $27(73)$ & $47(98)$ & $21(68)$ \\
\hline$* 1 / * 2$ & $11(18)$ & $18(22)^{\#}$ & $17(14)$ & $7(19)$ & $0(0)$ & $4(13)$ \\
\hline$\star 1 / * 3$ & $8(14)$ & $7(8)^{\#}$ & $17(14)$ & $3(8)$ & $1(2)$ & $4(13)$ \\
\hline$* 2 / * 2$ & $1(2)$ & $3(4)^{\#}$ & $1(1)$ & $0(0)$ & $0(0)$ & $1(3)$ \\
\hline$* 2 / * 3$ & $0(0)$ & $1(1)^{\#}$ & $1(1)$ & $0(0)$ & $0(0)$ & $1(3)$ \\
\hline$\star 3 / * 3$ & $0(0)$ & $1(1)^{\#}$ & $0(0)$ & $0(0)$ & $0(0)$ & $0(0)$ \\
\hline \multicolumn{7}{|l|}{ VKORC1 genotype", n (\%) } \\
\hline$-1639 \mathrm{G} / \mathrm{G}$ or $1173 \mathrm{C} / \mathrm{C}$ & $27(46)$ & $25(30)$ & $43(36)$ & $10(27)$ & $1(2)$ & $13(42)$ \\
\hline$-1639 \mathrm{~A} / \mathrm{G}$ or $1173 \mathrm{~T} / \mathrm{C}$ & $25(42)$ & $43(52)$ & $55(46)$ & $17(46)$ & $9(19)$ & $13(42)$ \\
\hline -1639A/A or $1173 T / T$ & $7(12)$ & $15(18)$ & $22(18)$ & $10(27)$ & $38(79)$ & $5(16)$ \\
\hline \multicolumn{7}{|l|}{ Ethnic origin } \\
\hline White (\%) & 100 & Not recorded but & 76 & 73 & & 77 \\
\hline Asian (\%) & & $\begin{array}{l}\text { mainly Caucasian } \\
\text { study subjects\#\# }\end{array}$ & 13 & 8 & 100 & 13 \\
\hline Black (\%) & & & 5 & 19 & & 6 \\
\hline Other (\%) & & & 6 & & & 3 \\
\hline \multicolumn{7}{|l|}{ Treatment related } \\
\hline $\begin{array}{l}\text { Indication for warfarin } \\
\text { therapy }\end{array}$ & Thrombosis & Cardiac & Mainly cardiac & Cardiac & Cardiac & Cardiac \\
\hline Target INR (\%) & $2.0-3.0(100)$ & $\begin{array}{l}1.5-3.3(37) \\
1.8-3.2(46) \\
2.5-4.0(17)\end{array}$ & $\begin{array}{l}2.0-3.0(84) \\
2.5-3-5(16)\end{array}$ & $\begin{array}{l}1.5-2.5(11) ; \\
2.0-3.0(51) \\
2.5-3.5(22) \\
3.0-4.0(16)\end{array}$ & $\begin{array}{l}\text { Not specified } \\
\text { (observed INR } \\
\text { range: } \\
1.1-3.3 \text { ) }\end{array}$ & $\begin{array}{l}1.7-3.0(10) \\
2.0-3.0(90)\end{array}$ \\
\hline Observed daily dose, in mg/kg & $0.03-0.6$ & $0.03-0.5$ & $0.02-0.43$ & $0.04-0.34$ & $0.04-0.21$ & $0.04-0.29$ \\
\hline Observed daily dose, in mg & & & & & & $0.56-9.11$ \\
\hline \multicolumn{7}{|c|}{ 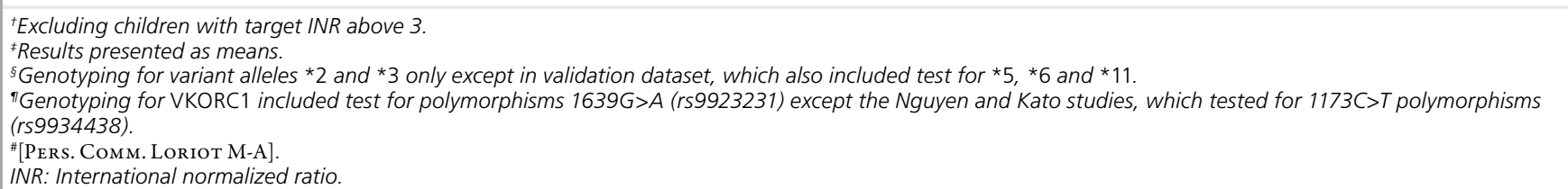 } \\
\hline
\end{tabular}

model, examining scatter plots of observed versus predicted values gives more information about the performance across the whole range of outcome values [18]. Other measures commonly used in the evaluation of warfarin models include the mean absolute error [32], or the clinical accuracy of model predictions [7] in which the proportion of patients with an ideal (within $\pm 20 \%$ of actual dose), overestimated (>20\% above actual dose) or underestimated (>20\% below actual dose) dose prediction are calculated. Rarely reported important measures of a warfarin model's predictive performance include bias (mean prediction error) and 
imprecision (mean squared prediction error). Bias is when there is a systematic difference between the predicted and the true value, and imprecision is the reproducibility, or scatter, of the predictions. Figure 2 provides an illustration of bias and imprecision.

\section{PK/PD-based models}

The same metrics that are used for linear regression models can be applied when evaluating $\mathrm{PK} / \mathrm{PD}$-based prediction models, including bias, imprecision and coefficient of determination. However, these measures are often complemented with simulation-based diagnostics such as the visual predictive check (VPC). The principle of a VPC is to graphically assess whether simulations from the model are able to reproduce both the central trend (the median curve) and the variability (outer percentiles) in the observed data when plotted against an independent variable (e.g., time, dose or age). A prediction corrected VPC is a special type of VPC that is appropriate for individually titrated drugs [33], such as warfarin.

\section{Evaluations of published prediction models in children Linear regression models}

The variability in dose explained by the four published linear regression models that included VKORC1 and CYP2C9 [14-17] varied from 38 [14] to $82 \%$ [16], with $V K O R C 1$ and CYP2C9 genotype explaining from 3.7 [14] to $47 \%$ [16] and 0.4 [14] to $12.8 \%$ [17], respectively. Two out of five studies investigated but found no association between CYP4F2 genotype and warfarin dose $[16,18]$. Detailed results for all regression models are summarized in Table 2 . Based on 34 children with thrombosis, Nowak-Göttl et al. [14] concluded that age was the most important determinant, accounting for $28.3 \%$ of the dose variability. Kato et al. reported that $V K O R C 1$ genotype and age were the main factors influencing warfarin dosing in a cohort of 48 Japanese children with heart disease [28]. In the study by Moreau et al. on 83 children with heart disease, height was the single most important predictor, explaining $48.1 \%$ of the dose variability [15]. In the largest study, including 120

Table 2. Published linear regression models for warfarin dosing in children, with information on individual predictors and their relative contribution in explaining dose variability.

\begin{tabular}{|c|c|c|c|c|c|}
\hline $\begin{array}{l}\text { Dose variability, } \\
\text { explained by \% }\end{array}$ & Nowak-Göttl ${ }^{\dagger}$ et al. [14] & Biss $^{\ddagger}$ et al. $[17]$ & Moreau $^{\S}$ et al. [15] & Nguyen" et al. [16] & Kato\# et al. [28] \\
\hline $\begin{array}{l}\text { Units dose was } \\
\text { predicted in }\end{array}$ & $\mathrm{mg} / \mathrm{kg} /$ day & mg/day & mg/week & $\mathrm{mg} / \mathrm{kg} /$ day & mg/day \\
\hline \multicolumn{6}{|l|}{ Genetic factors } \\
\hline CYP2C9 genotype & $0.4 \%$ & $12.8 \%$ & $2.0 \%{ }^{+\dagger}$ & $5.0 \%{ }^{\dagger+}$ & \\
\hline VKORC1 genotype & $3.7 \%$ & $26.6 \%$ & $18.2 \%{ }^{\dagger+}$ & $47 \%^{++}$ & $X^{\ddagger \ddagger}$ \\
\hline CYP4F2 genotype & & No effect & No effect & & \\
\hline \multicolumn{6}{|l|}{ Demographics } \\
\hline Age (years) & $28.3 \%$ & & & $12 \%^{++}$ & $X^{\ddagger \ddagger}$ \\
\hline Weight (kg) & $X^{\ddagger \ddagger}$ & & & $X^{\ddagger \ddagger}$ & $X^{\ddagger \ddagger}$ \\
\hline Height (cm) & & $29.8 \%$ & $48.1 \%^{+\dagger}$ & & \\
\hline \multicolumn{6}{|l|}{ Treatment related } \\
\hline Indication & & $3.2 \%$ & & No effect & \\
\hline Target INR & No effect & No effect & $4.4 \%^{t+}$ & $18 \%^{+\dagger}$ & $X^{\ddagger \ddagger}$ \\
\hline \multicolumn{6}{|l|}{ Full model } \\
\hline All predictors & $38 \%$ & $72 \%$ & $70 \%$ & $82 \%$ & \\
\hline \multicolumn{6}{|c|}{ 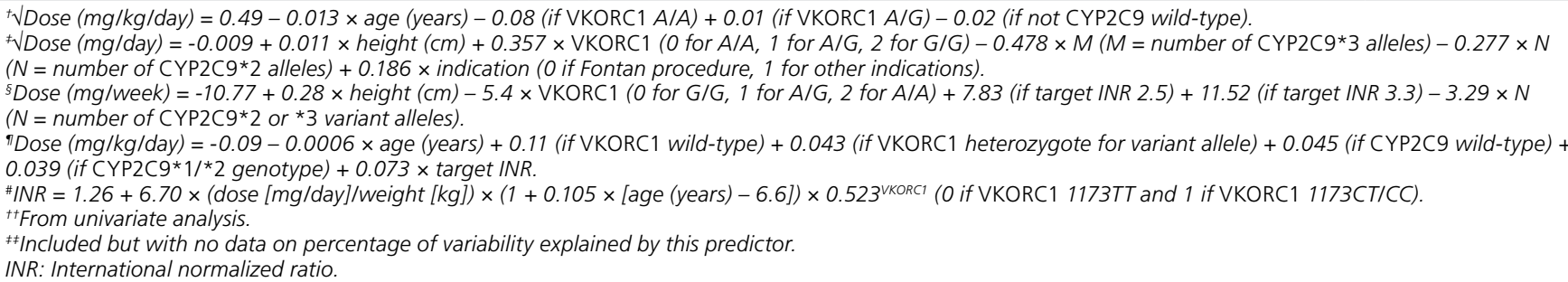 } \\
\hline
\end{tabular}




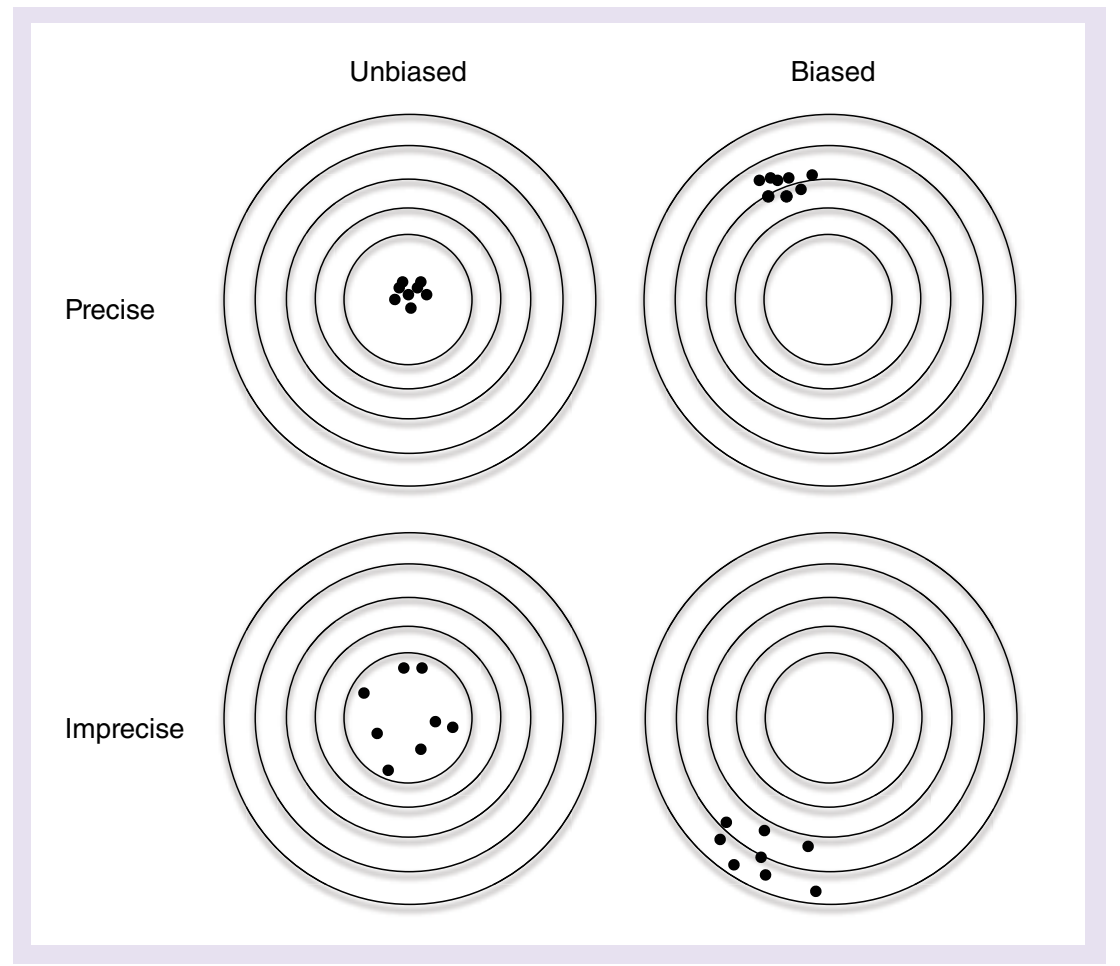

Figure 2. Measures of accuracy (reported as bias) and precision (reported as imprecision) of model predictions. The bull's eye represents the true dose. A prediction model with systematic errors provides dose predictions that are biased, and a prediction model with large random errors provides dose predictions that are imprecise or scattered. The top left figure represent predictions that are both precise and unbiased whereas the bottom right figure represent predictions that are both imprecise and biased.
The model by Lala et al. was evaluated in an external cohort of 26 children (mean age: 4.4 years) [29]. The authors used simulationbased diagnostics to compare model predicted and observed INR profiles, and concluded that model performance was reasonable, with no particular genotype having a higher probability of failing the validation [29]. However, it is worth noting that 22 of 26 children carried the CYP2C9 wild-type genotype $\left({ }^{*} 1 /{ }^{*} 1\right)$, while the remaining four children were heterozygous for the ${ }^{*} 2$ variant allele, and no-one carried ${ }^{*} 3$.

\section{Head-to-head comparison of prediction models for children}

A subset of a published pediatric dataset [27] was used to compare accuracy in maintenance dose predictions between six of the published pharmacogenetics-based dosing algorithms for children [14-17,27,29]. The model by Kato et al. was excluded since it was developed in Japanese children with a very low frequency of $C Y P 2 C 9$ variant alleles, and did not include CYP2C9 genotype as a predictor for warfarin dose [28]. The validation dataset consisted of data from children who fulfilled the following criteria: had complete information for all predictors included in at least one of the prediction models; target INR within the range 1.7-3.0; and the observed warfarin dose represented the average daily maintenance dose from a period of stable warfarin therapy defined as: having at least three consecutive INRs within target range over a minimum period of 4 weeks, and with no change in dose during this time period. Thirty-one children, with a median age of 3.2 years (range: $0.33-15.6$ years) fulfilled all criteria and were included in the model comparison. Patient characteristics for this cohort are included in TABLE 1 in the far right column. The median observed maintenance dose in this cohort was $2.32 \mathrm{mg}$, or $0.14 \mathrm{mg} / \mathrm{kg}$. Dose predictions for the four regression models were performed in Microsoft Excel, Version 12.3.2, using the equations as presented in the footnote of Table 2. Dose predictions with Hamberg's model were performed in NONMEM using the approach by Jönsson and Karlsson [34], and for the Lala model, individual dose predictions were derived using the optimized starting dose as provided in Table 2 of their original paper [29]. The output from each algorithm was converted, if applicable, to a predicted daily dose in $\mathrm{mg}$, and compared with the actual maintenance dose. Each model was evaluated in the same external dataset by generating plots of observed versus model predicted 
dose (including $\mathrm{R}^{2}$ values), and results on bias, imprecision and clinical accuracy of individual model predictions.

Figure 3 shows scatter plots of observed versus model predicted maintenance dose for the six models. The two dotted lines in each plot represent dose predictions that were $\pm 20 \%$ of the observed maintenance dose (ideal dose predictions). Points above the upper dotted line represent dose predictions that were underestimated with $>20 \%$, and points below the lower dotted line represent dose predictions that were overestimated with $>20 \%$. Each plot also includes a regression line of observed versus predicted dose and its coefficient of determination $\left(\mathrm{R}^{2}\right)$. Results on bias, imprecision and clinical accuracy are presented in TABLE 3. The three models with the highest coefficient of determination, Nguyen et al. [16], Lala et al. [29] and Hamberg et al. [27], had $\mathrm{R}^{2}$ values ranging from 0.764 to 0.777 . However, the Nguyen model [16] showed signs of systematic overpredictions, with a positive bias of $1.27 \mathrm{mg}$. Also the Lala model [29] showed signs of bias, with doses systematically underpredicted with $1.18 \mathrm{mg}$. Overall, when considering all measures of predictive performance $\left(\mathrm{R}^{2}\right.$, bias, imprecision and clinical accuracy), the model by Hamberg et al. [27] gave the most accurate dose predictions, while the linear regression model by Biss et al. [17] was the second best. When the Hamberg model was used for a posteriori dose predictions, with inclusion of up to three INR observations, model predictions were further improved, with bias reduced from -0.39 to $-0.06 \mathrm{mg}$, and imprecision from 0.99 to $0.67 \mathrm{mg}$, while the percentage of ideal dose predictions increased from 45 to $74 \%$, and the coefficient of determination increased from 0.777 to 0.880 .

\section{Discussion}

There is a paucity of studies investigating pharmacogenetics and other causes of variability in warfarin response in children. The studies reported to date have been limited by sample size, including only 34 to 120 children per study. In comparison, several adult dose prediction models [6-8] have been developed on sample sizes well above a thousand patients, and in addition on more homogeneous patient populations. Population PK/PD models can alleviate many of the difficulties encountered when developing dosing regimens for small patient populations, for example, children. Models that have been developed on adult data are increasingly being used to support dosing in children, and this approach is strongly advocated by regulatory bodies such as the FDA and EMA $[35,36]$. The possibility to extrapolate knowledge from adults to children is based on pharmacological principles that acknowledge that adults and children are in some aspects similar and not discrete populations. In our head-to-head comparison of prediction models, four out of six models were derived from studies conducted in children [14-17] and the others were theoretically bridged to children from models developed in adults $[27,29]$. Out of all the models, the bridged model by Hamberg et al. [27] gave the most accurate dose predictions, and the model by Biss et al. was second best. When considering only models derived from studies in children, the model by Biss et al. [17], which was derived from the largest study with data from 120 children, showed the best predictive properties.

A common way to present the importance of individual factors on warfarin dose variability is by reporting the percentage of variability explained by a given factor. It provides a measure of what factors are important for the total variability on a population level, and this is influenced by both the effect size of the individual factor and of how common this factor is in the study population. However, when evaluating predictors important for dose individualization, the clinical validity of this measure may be questioned. For the individual patient, a more relevant measure would be how much the dose can be influenced by a given factor. From the studies conducted in children, CYP2C9 genotype was reported to explain from 0.4 [14] to $12.8 \%$ [17], and VKORC1 genotype from 3.7 [14] to $47 \%$ [16] of the variability in warfarin dose. The large differences in dose variability explained by genotype may be owing to study differences in minor allele frequencies [37], and grouping of $C Y P 2 C 9$ genotype effects combined with the small sample sizes, which may result in point estimates that are biased and associated with large uncertainty. Analyses of large cohorts of adult warfarin patients have shown that the proportion of variability in dose explained by VKORC1 genotype was higher in white patients than in black or Asian patients, and that this was largely explained by large inter-racial differences in allele frequencies [37]. Although at a population level the contribution of VKORC1 genotype on dose requirements is higher in white patients, CYP2C9 can be more important on an individual level. When looking at individual patients, studies in adults have shown that 

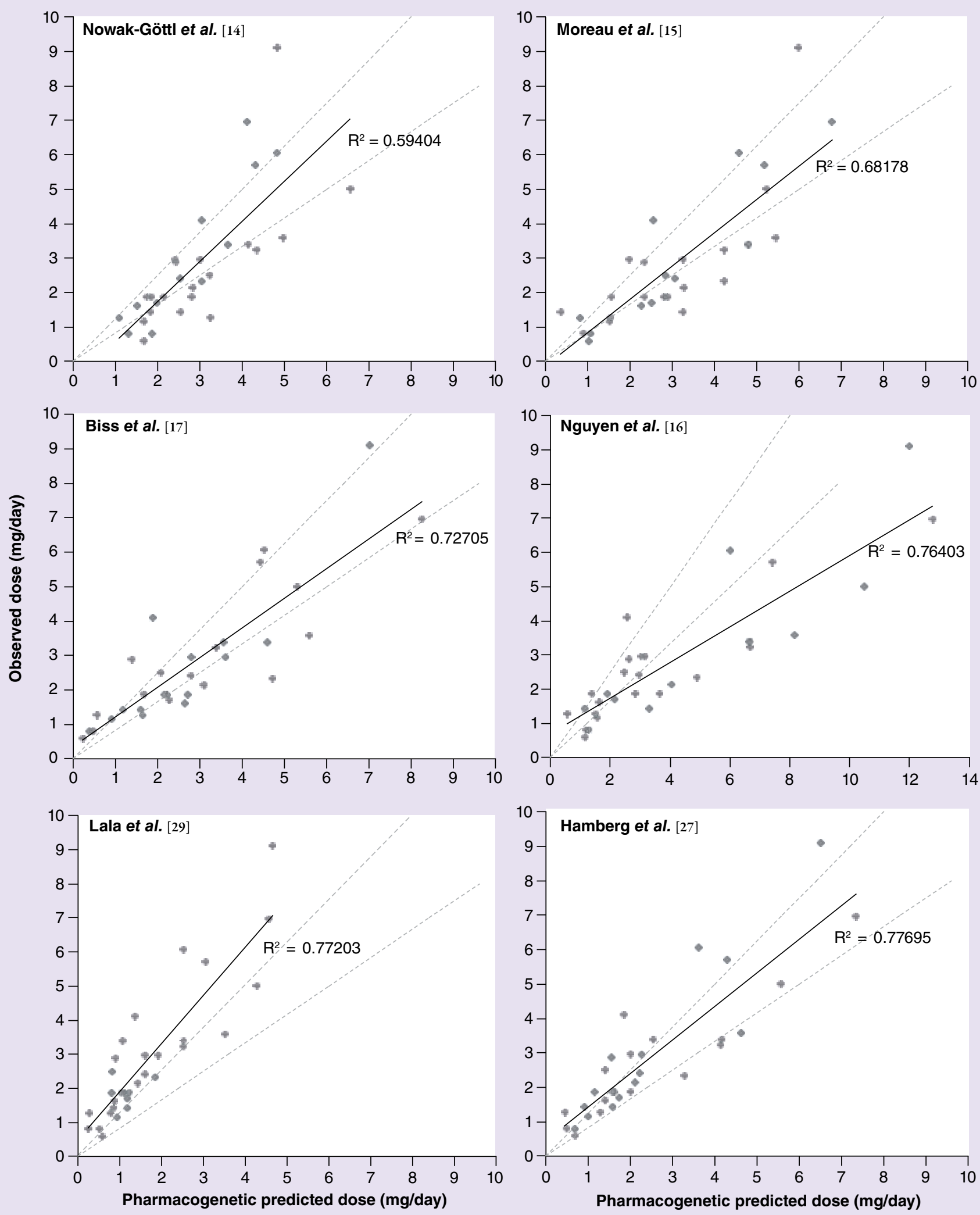

Figure 3. Observed versus predicted maintenance dose $(\mathrm{mg} / \mathrm{day})$ for the linear regression models and the bridged pharmacokinetic/pharmacodynamic models evaluated in a cohort of 31 independent warfarin-treated children with a target international normalized ratio in the range 1.7-3.0. The linear regressions models are by Nowak-Göttl et al. [14], Moreau et al. [15], Nguyen et al. [16] and Biss et al. [17] and the bridged pharmacokinetic/pharmacodynamic models by Hamberg et al. [27] and Lala et al. [29]. Points between the two dotted lines represent predicted doses within $\pm 20 \%$ of observed doses, with points above the upper dotted line representing doses that were under predicted with $>20 \%$ and points below the lower dotted line representing doses that were over predicted with $>20 \%$. The coefficient of determination $\left(R^{2}\right)$ is included for individual algorithms as a measure of overall model performance. 
Table 3. Percentage of children with predicted maintenance dose underpredicted (prediction $<80 \%$ of actual dose), ideal (prediction $\pm 20 \%$ of actual dose) or overpredicted (prediction $>120 \%$ of actual dose).

\begin{tabular}{|c|c|c|c|c|c|c|}
\hline $\begin{array}{l}\text { Prediction model } \\
\text { by }\end{array}$ & Ref. & $\begin{array}{l}\text { Underpredicted } \\
\text { (\%) }\end{array}$ & $\begin{array}{l}\text { Ideal } \\
(\%)\end{array}$ & $\begin{array}{l}\text { Overpredicted } \\
\text { (\%) }\end{array}$ & Bias & Imprecision \\
\hline Nowak-Göttl et al. & {$[14]$} & 16 & 35 & 48 & 0.10 & 1.25 \\
\hline Moreau et al. & {$[15]$} & 19 & 26 & 55 & 0.23 & 1.11 \\
\hline Biss et al. & {$[17]$} & 32 & 39 & 29 & 0.04 & 1.04 \\
\hline Nguyen et al. & {$[16]$} & 10 & 26 & 65 & 1.27 & 2.21 \\
\hline Lala et al. & [29] & 87 & 13 & 0 & -1.18 & 1.58 \\
\hline Hamberg et al. & [27] & 42 & 45 & 13 & -0.39 & 0.99 \\
\hline
\end{tabular}

polymorphisms in $C Y P 2 C 9$ can cause up to a 4.2 -fold difference in dose $\left(C Y P 2 C 9^{*} 3 /{ }^{*} 3\right.$ vs $\left.{ }^{*} 1 /{ }^{*} 1\right)$ while polymorphisms in $\operatorname{VKORC1}$ only can cause up to a twofold difference in dose (VKORC1 A/A vs G/G) [23].

When scaling adult doses to children, linear predictions of dose based on bodyweight are commonly used, and this is the case for three of the prediction models reviewed here [14,16,29]. However, it is becoming widely recognized that the relationship between adult and pediatric doses is nonlinear. Owing to a larger liver and/or kidneys in relation to body size, small children often require a higher mg dose per kilo bodyweight than older children and adults to achieve the same drug exposure [30]. This has also been observed for warfarin where higher weight-normalized doses have been reported in the youngest age groups to obtain the same target INR $[17,28,38]$. The prediction models by Hamberg et al. [27] and Lala et al. [29] accommodate the nonlinear relationship between warfarin dose and bodyweight by using commonly accepted allometric scaling methods [30,39]. However, the Lala dosage scheme was simplified to provide two distinct $\mathrm{mg} / \mathrm{kg}$ doses within each genotype combination, one for larger $(\geq 20 \mathrm{~kg})$ and one for smaller $(<20 \mathrm{~kg})$ bodyweights [29].

There is a certain degree of understanding of the effect of clinical, demographic and genetic factors on the variability in warfarin dose, but less on how these factors influence the variability in rate of response. Examples of temporal outcomes of interest in warfarin therapy include the time delay between drug exposure and INR response, the extent and rate of drug accumulation and the time to stable treatment response (steady state) after starting warfarin therapy or after dose adjustments. PK/PD models can provide a better understanding of the dose-response relationship over time, and are able to acknowledge variability in both rate and extent of response and translate this into individualized dosing regimens. A commonly used warfarin dose revision algorithm for children [2] assumes a similar rate of response in all patients. To illustrate the importance of rate of response, we describe four examples with children with different $C Y P 2 C 9$ or VKORC1 genotype combinations. Figure 4 illustrates the time course of predicted INR response and amount of $S$-warfarin in the body for each child, after receiving daily doses of an individualized maintenance dose. Example A includes two children with the VKORC1 genotype G/G but with different CYP2C9 genotypes; one with ${ }^{*} 1{ }^{*} 1$ and one with ${ }^{*} 3 /{ }^{*} 3$. Example B includes two children with the CYP2C9 genotype ${ }^{*} 1 /^{*} 1$ but with different $V K O R C 1$ genotypes; one with G/G and one with A/A. Of note here is the difference in time to a therapeutic INR $(\geq 2)$ and a stable INR (2.5) in example A. The child with CYP2C9*3/*3 genotype has a low metabolic capacity, and a longer elimination half-life $\left(\mathrm{t}_{1 / 2}\right)$, which leads to more drug accumulation and a longer time to steady state and stable INR compared with the child with $C Y P 2 C 9^{*} 1{ }^{*} 1$ genotype. Differences in $V K O R C 1$ genotype, on the other hand, influence the sensitivity to warfarin but not the rate of response, as illustrated in example B.

The Hamberg model showed a tendency to overpredict the INR response, or conversely underpredict the dose, in children 2 years old or younger [27]. This may be due to inaccurate scaling of the pharmacokinetics of warfarin in this age group, that is, underestimating clearance and overestimating warfarin exposure. It could also be due to differences in pharmacodynamics, for example, that young children are 
less sensitive to warfarin than older children or adults. Another possibility is that it is caused by the practical aspects of administering warfarin to young children, which often involves the crushing of tablets and mixing with food or liquids. This makes accurate and reproducible dosing difficult and could lead to overestimation of the actual dose delivered. In principle, irrespective of the cause, model predictions could be improved by optimizing the model on data from warfarin-treated children, preferably using a dataset enriched with treatment data from young children and children with rare genotypes.

An advantage when analyzing study data using population modeling is that longitudinal treatment data from the same subject can be included in the analysis, irrespective of when the data was collected (at the start of therapy, during stable anticoagulation, following dose revisions). To get accurate and reliable results it is imperative that the dose history is complete and correct, but this holds true regardless of the method used to analyze the data. In the majority of the published warfarin studies, a single observation from the time of stable anticoagulation is used in the analysis, which may introduce a selection bias by excluding unstable patients that are difficult to treat. Furthermore, to be able to investigate the effect of growth and maturation on the response to drugs in neonates and young children, access to longitudinal treatment data is a prerequisite.

A practical limitation of a PK/PD model is that it is not as easy to implement as a linear regression model that consists of a simple equation that can be implemented in, for example, Excel. A PK/PD model often consists of a set of differential equations without a closed form solution and needs a differential equation solver to operate. To address this, the Hamberg NONMEM model [27] has been transferred to a new Java-based tool for a priori and a posteriori dose predictions in both children and adults [104]. Besides dose predictions, the tool can be used for prediction of early INR response for a given dose regimen. This could help identify situations where warfarin therapy could be improved, for example, with administration of loading doses and/or delayed INR monitoring or dose adjustments.

The clinical utility of genotype-guided dose initiation has been addressed in two recently published warfarin trials in adults, the EU-PACT trial [40] and the COAG trial [41]. The EU-PACT trial found genotype-guided dosing to be superior to standard dosing with respect to both the primary outcome measure, with a $7 \%$ increase in time in therapeutic range (\%TTR) over the first 12 weeks of therapy, and on several secondary outcome measures, for example, a 69\% reduction in INR $>4$ and a $28 \%$ reduction in the time to the therapeutic INR [40]. On the other hand, the COAG trial found no benefit of genotypeguided over clinically guided dose initiation on the primary outcome, which was \%TTR from day $4 / 5$ through to day 28 [41]. A limitation of the study design of both studies was that dosing in the test and reference arms were identical after the initial 3-5 days of genotype-guided dosing. The EU-PACT trial dosed according to usual clinical practice [40], while the COAG trial used standardized dose-adjustment techniques [41]. It may be argued that dose revision in the test arm should have been informed by genotyping for more than 5 days to fully explore the potential benefit of genotype-based dosing. Especially when considering results from the large RE-LY study, which suggest that dose adjustment practices and support systems are the main factors defining the quality of anticoagulation therapy, as measured by \%TTR [42].

Several properties are desirable for an optimal prediction model for warfarin dosing in children. Preferably a single model should be able to predict an individualized initiation dose (a priori predictions) and to provide individualized dose revisions (a posteriori predictions). The model should be able to operate both with and without information on important predictors, for example, genotype. The model should also be flexible and handle variability in both baseline and target INR between patients and populations. The model should be able to interpret variability in both rate and extent of response and to translate this into individualized dosing recommendations. Finally, the model should be flexible so that new knowledge that emerges over time could be easily incorporated.

\section{Conclusion}

To demonstrate the utility of a specific prediction model in clinical practice, well-designed prospective, randomized trials are needed to compare model-based dosing against standardof-care. This is probably best done as a multicenter trial that involves centers from all over the world. This would increase the sample size, and increase the geographic and ethnic diversity of the study population, hence increasing the generalizability of study results. 


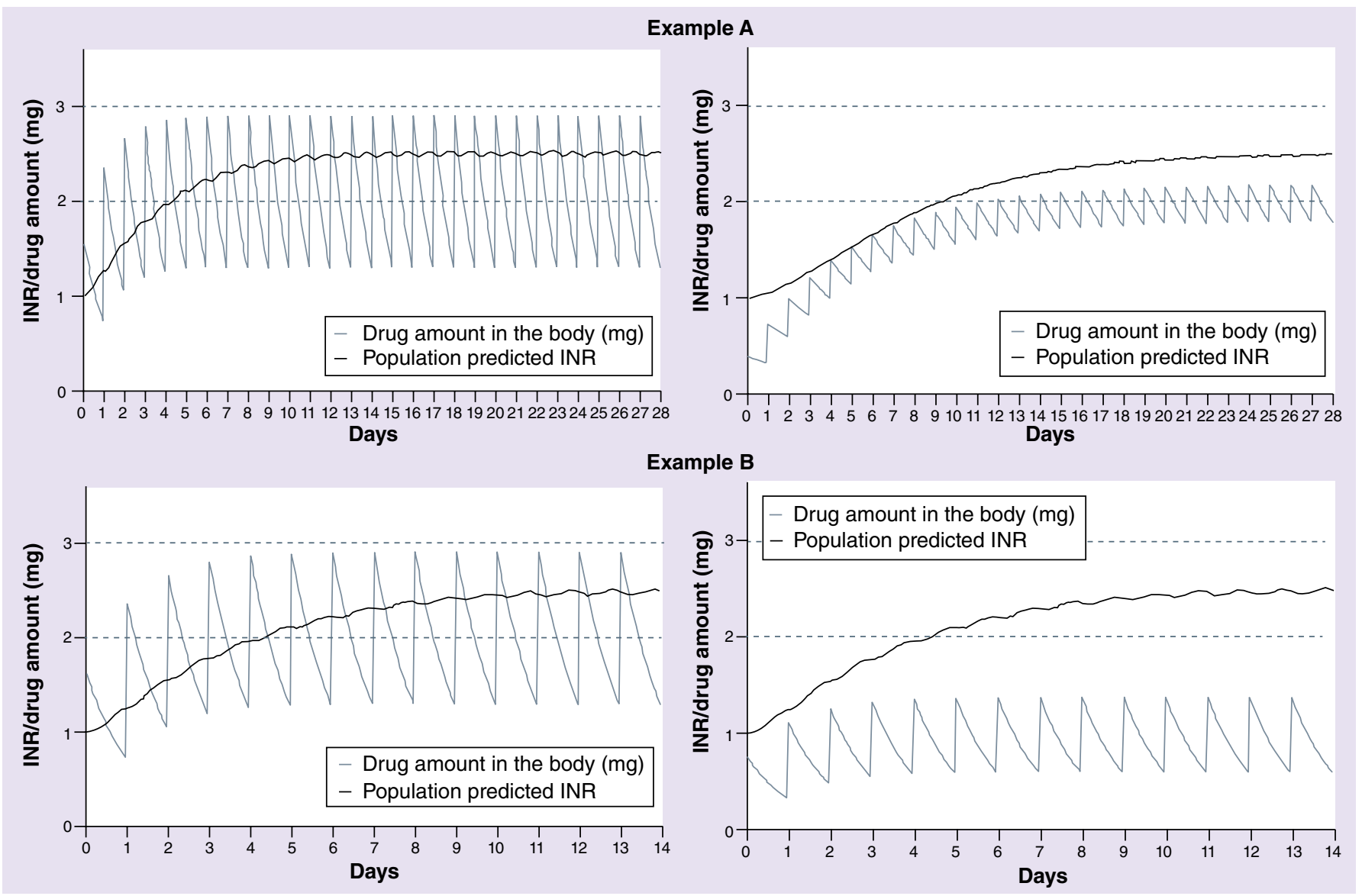

Figure 4. Model predicted international normalized ratio response (black) and amount of $S$-warfarin in the body (gray) over time for typical children with different genotype combinations. The dotted lines represent the target INR range of 2-3. Example A includes a child with the genotype combination CYP2C9*1/*1 and VKORC1 G/G (left) and a child with the genotype combination CYP2C9*3/*3 and VKORC1 G/G (right), and Example B includes a child with the genotype combination CYP2C9*1/*1 and VKORC1 G/G (left), and a child with the genotype combination CYP2C9*1/*1 and VKORC1 A/A (right). Simulations are performed using the pharmacokinetic/pharmacodynamic-based warfarin model by Hamberg et al. [27].

INR: International normalized ratio.

\section{Future perspective}

Although the predictive performance of the bridged warfarin model by Hamberg et al. [27] gives some promise, there are still knowledge gaps that needs to be filled to optimize dose predictions in children of all ages. There are a number of issues that cannot be bridged from studies conducted in adults and that need to be addressed in dedicated pediatric studies. Some of these include:

- Variability in delivered dose due to lack of a flexible and customized pediatric formulation. Warfarin dosing in young children often involves actions that precludes accurate and reproducible dosing [43], such as splitting and crushing of tablets, and mixing with food or liquids before administration;

- The impact of dietary differences. This is especially important in the youngest age groups, as breast milk and infant formulas have very different vitamin $\mathrm{K}$ content [43]. In a study by Streif et al. [44] it was shown that children receiving vitamin $K$ enriched enteral nutrition, required significantly higher warfarin doses $(0.26 \pm 0.18 \mathrm{mg} / \mathrm{kg})$ to maintain INR within target compared with children not receiving enteral nutrition $(0.11 \pm 0.7 \mathrm{mg} / \mathrm{kg})$;

- The influence of age and body size on warfarin dose variability in children. Age, weight and height are often highly correlated in children, and are sometimes used interchangeably. However, from a biological standpoint they represent two distinct aspects. Weight and height in children represent the expected growth (change in size) of a child over time, whereas age represents the expected maturation (change in activity) of biological systems over time, for example, the maturation of metabolizing enzymes. When studying maturation in neonates, especially in premature children, it is important to consider both 
postmenstrual age or gestational age, and postnatal age;

- Improved understanding of the maturation (ontogeny) of the CYP2C9 and VKORC1 enzymes. Pharmacogenetic variability is superimposed upon developmental patterns of enzyme expression (ontogeny) in young children, thereby adding a dimension of complexity that is not present in adults [45]. Studies have shown that genotype-phenotype relationships can change in developing children, with genotype effects only being evident when the gene is mature and the enzyme is expressed;

- The influence of intercurrent illnesses and concurrent medications on warfarin dose variability in children. The type of intercurrent illnesses and concurrent medications are often different in children compared with adult warfarin patients, and also varies with age [44];

- Identification of optimal INR therapeutic ranges in children. The therapeutic ranges that are used in children today are extrapolated from studies in adults. No studies have validated these ranges against clinical outcome data (bleeding or clot) for the various indications in children or neonates [43].

Some of these issues could be addressed by performing meta-analyses of existing studies via an international pediatric warfarin consortium, similar to what has been established for adults [7]. Using population PK/PD models when analyzing treatment data from longitudinal studies enables estimation of quantitative and temporal parameters associated with the metabolism- and concentration-dependent response to warfarin, and provides the necessary context for interpreting INR measurements from all phases of therapy [24]. These types of models can individualize starting doses, and more importantly, ensure consistent and more efficient dose revision practices through the use of Bayesian forecasting methods for warfarin dose individualization in children. With improved support systems that ensure consistent and efficient dose adjustment practices, it may even be possible to identify specific target INR ranges in children.

\section{Financial \& competing interests disclosure}

A-K Hamberg is supported by a personal grant from the Ränk family via the Swedish Heart and Lung Foundation. $M$ Wadelius is supported by the Swedish Research Council (Medicine 523-2008-5568 and 521-2011-2440), the Swedish Heart and Lung Foundation, the Clinical Research Support (ALF) at Uppsala University. The funding organizations played no role in the writing of this review. The authors have no other relevant affiliations or financial involvement with any organization or entity with a financial interest in or financial conflict with the subject matter or materials discussed in the manuscript apart from those disclosed.

No writing assistance was utilized in the production of this manuscript.

\section{Executive summary}

\section{Warfarin dose prediction models}

- Warfarin is the most commonly used anticoagulant for prevention and treatment of thromboembolic events in both adults and children.

- Therapy is complicated by a narrow therapeutic range and large variability in dose required for adequate effect.

" A too high dose gives an increased risk of bleeding, and a too low dose gives an increased risk of clotting.

Studies in adults have shown that variations in two genes, CYP2C9 and VKORC1, contribute to the variability in warfarin dose.

" This knowledge has been used to develop prediction models that provide individualized dosing of warfarin in adults.

" Less is known about the impact of genotype and other factors on dose variability in children.

\section{Evaluation of prediction models}

This article reviews and evaluates published pharmacogenetic-based prediction models for warfarin dosing in children.

A head-to-head comparison of published models found that a pharmacokinetic/pharmacodynamic-model bridged to children provided the most accurate dose predictions in children.

- The prediction model includes CYP2C9 and VKORC1 genotype, age, bodyweight, target international normalized ratio (INR) and baseline INR as predictors of warfarin dose.

- A prediction model for warfarin dosing in children should predict both an initiation dose (a priori dose predictions) and subsequent dose revisions (a posteriori dose predictions), should operate both with and without information on important predictors, should manage differences in both baseline INR and target INR, and should accommodate variability in both rate and extent of response to optimize warfarin dosing in individual patients.

- The clinical utility of a new prediction model should be demonstrated in well-designed, prospective, randomized trials against standard-of-care. 


\section{Open Access}

This work is licensed under the Creative Commons Attribution-NonCommercial 3.0 Unported License. To view a copy of this license, visit http:// creativecommons.org/licenses/by-nc-nd/3.0/

\section{References}

Papers of special note have been highlighted as: - of interest

" " of considerable interest

1 Jorgensen AL, FitzGerald RJ, Oyee J, Pirmohamed M, Williamson PR. Influence of CYP2C9 and $\mathrm{VKORC1}$ on patient response to warfarin: a systematic review and metaanalysis. PLoS ONE 7(8), e44064 (2012).

2 Monagle P, Chan AKC, Goldenberg NA et al. Antithrombotic therapy in neonates and children: antithrombotic therapy and prevention of thrombosis (9th Edition): American College of Chest Physicians evidence-based clinical practice guidelines. Chest 141(2 Suppl.), e737S-e801S (2012).

3 Hamberg AK, Dahl ML, Barban M et al. A PK-PD model for predicting the impact of age, CYP2C9, and VKORC1 genotype on individualization of warfarin therapy. Clin. Pharmacol. Ther. 81(4), 529-538 (2007).

- First population pharmacokinetic (PK)/pharmacodynamic (PD) model of $S$ and $R$-warfarin that investigated the influence of CYP2C9 and VKORC1 genotype on warfarin's $P K$ and $P D$.

4 Sconce EA. The impact of CYP2C9 and $V K O R C 1$ genetic polymorphism and patient characteristics upon warfarin dose requirements: proposal for a new dosing regimen. Blood 106(7), 2329-2333 (2005).

5 Millican EA, Lenzini PA, Milligan PE et al. Genetic-based dosing in orthopedic patients beginning warfarin therapy. Blood 110(5), 1511-1515 (2007).

6 Gage B, Eby C, Johnson J et al. Use of pharmacogenetic and clinical factors to predict the therapeutic dose of warfarin. Clin. Pharmacol. Ther. 84(3), 326-331 (2008).

7 International Warfarin Pharmacogenetics Consortium, Klein TE, Altman RB et al. Estimation of the warfarin dose with clinical and pharmacogenetic data. N. Engl. J. Med. 360(8), 753-764 (2009).

8 Wadelius M, Chen LY, Lindh JD et al. The largest prospective warfarin-treated cohort supports genetic forecasting. Blood 113(4), 784-792 (2009).

9 Biss TT, Avery P, Williams MD, Brandao LR, Grainger JD, Kamali F. The VKORC1 and CYP2C9 genotypes are associated with overanticoagulation during initiation of warfarin therapy in children. J. Thromb. Haemost. 11, 373-375 (2013).
10 Lindh J, Lundgren S, Holm L, Alfredsson L, Rane A. Several-fold increase in risk of overanticoagulation by $C Y P 2 C 9$ mutations. Clin. Pharmacol. Ther. 78(5), 540-550 (2005).

11 Limdi N, McGwin G, Goldstein J et al. Influence of CYP2C9 and VKORC1 $1173 \mathrm{C} / \mathrm{T}$ genotype on the risk of hemorrhagic complications in African-American and European-American patients on warfarin. Clin. Pharmacol. Ther. 83(2), 312-321 (2007).

12 Lindh JD, Holm L, Dahl ML, Alfredsson L, Rane A. Incidence and predictors of severe bleeding during warfarin treatment. J. Thromb. Thrombolysis 25(2), 151-159 (2007).

13 Meckley LM, Wittkowsky AK, Rieder MJ, Rettie AE, Veenstra DL. An analysis of the relative effects of $V K O R C 1$ and $C Y P 2 C 9$ variants on anticoagulation related outcomes in warfarin-treated patients. Thromb. Haemost. 100 (2), 229-239 (2008).

14 Nowak-Göttl U, Dietrich K, Schaffranek D et al. In pediatric patients, age has more impact on dosing of vitamin $\mathrm{K}$ antagonists than VKORC1 or CYP2C9 genotypes. Blood 116(26), 6101-6105 (2010).

15 Moreau C, Bajolle F, Siguret V et al. Vitamin $\mathrm{K}$ antagonists in children with heart disease: height and $V K O R C 1$ genotype are the main determinants of the warfarin dose requirement. Blood 119(3), 861-867 (2012).

16 Nguyen N, Anley P, Yu MY, Zhang G, Thompson AA, Jennings LJ. Genetic and clinical determinants influencing warfarin dosing in children with heart disease. Pediatr. Cardiol. 34(4), 984-990 (2012).

17 Biss TT, Avery PJ, Brandao LR et al. VKORC1 and CYP2C9 genotype and patient characteristics explain a large proportion of the variability in warfarin dose requirement among children. Blood 119(3), 868-873 (2012).

"- Largest pediatric study to date used to derive a prediction model for children.

18 Eriksson N, Wadelius M. Prediction of warfarin dose: why, when and how? Pharmacogenomics 13(4), 429-440 (2012).

19 Lu Y, Yang J, Zhang H, Yang J. Prediction of warfarin maintenance dose in Han Chinese patients using a mechanistic model based on genetic and non-genetic factors. Clin. Pharmacokinet. 52(7), 567-581 (2013).

20 Avery P, Jorgensen A, Hamberg AK, Wadelius M, Pirmohamed M, Kamali F. A proposal for an individualized pharmacogenetics-based warfarin initiation dose regimen for patients commencing anticoagulation therapy. Clin. Pharmacol. Ther. 90(5), 701-706 (2011).
21 Lenzini P, Wadelius M, Kimmel S et al. Integration of genetic, clinical, and INR data to refine warfarin dosing. Clin. Pharmacol. Ther. 87(5), 572-578 (2010).

22 Mould DR, Upton RN. Basic concepts in population modeling, simulation, and model-based drug development. CPT: Pharmacometrics Syst. Pharmacol. 1(9), e6 (2012).

- Good introduction to the field of population PK/PD modeling.

23 Hamberg AK, Wadelius M, Lindh JD et al. A pharmacometric model describing the relationship between warfarin dose and INR response with respect to variations in CYP2C9, VKORC1, and age. Clin. Pharmacol. Ther. 87(6), 727-734 (2010).

24 Linder MW, Bon Homme M, Reynolds KK et al. Interactive modeling for ongoing utility of pharmacogenetic diagnostic testing: application for warfarin therapy. Clin. Chem. 55(10), 1861-1868 (2009).

25 Perlstein TS, Goldhaber SZ, Nelson K et al. The Creating an Optimal Warfarin Nomogram (CROWN) Study. Thromb. Haemost. 107(1), 59-68 (2012).

26 Holford NH. Clinical pharmacokinetics and pharmacodynamics of warfarin. Understanding the dose-effect relationship. Clin. Pharmacokinet. 11(6), 483-504 (1986).

27 Hamberg AK, Friberg LE, Hanséus K et al. Warfarin dose prediction in children using pharmacometric bridging - comparison with published pharmacogenetic dosing algorithms. Eur. J. Clin. Pharmacol. 69(6), 1275-1283 (2013).

"- A population PK/PD model for dose prediction in children. Showed the best predictive properties when compared with other prediction models for children.

28 Kato Y, Ichida F, Saito K et al. Effect of the VKORC1 genotype on warfarin dose requirements in Japanese pediatric patients. Drug Metab Pharmacokinet. 26(3), 295-299 (2011).

29 Lala M, Burckart GJ, Takao CM, Pravica V, Momper JD, Gobburu JVS. Genetics-based pediatric warfarin dosage regimen derived using pharmacometric bridging. J. Pediatr. Pharmacol. Ther. 18(3), 209-219 (2013).

30 Holford N. Dosing in children. Clin. Pharmacol. Ther. 87(3), 367-370 (2010).

- Good review on important aspects of dosing in children.

31 Johnson TN, Rostami-Hodjegan A, Tucker GT. Prediction of the clearance of eleven drugs and associated variability in neonates, infants and children. Clin. Pharmacokinet. 45(9), 931-956 (2006). 
32 Shaw PB, Donovan JL, Tran MT, Lemon SC, Burgwinkle P, Gore J. Accuracy assessment of pharmacogenetically predictive warfarin dosing algorithms in patients of an academic medical center anticoagulation clinic. J. Thromb. Thrombolysis 30(2), 220-225 (2010).

33 Bergstrand M, Hooker AC, Wallin JE, Karlsson MO. Prediction-corrected visual predictive checks for diagnosing nonlinear mixed-effects models. AAPS J. 13(2), 143-151 (2011).

34 Jönsson S, Karlsson MO. Estimation of dosing strategies aiming at maximizing utility or responder probability, using oxybutynin as an example drug. Eur. J. Pharm. Sci. 25(1), 123-132 (2005).

35 Manolis E, Pons G. Proposals for model-based paediatric medicinal development within the current European Union regulatory framework. Br. J. Clin. Pharmacol. 68(4), 493-501 (2009).

36 Manolis E, Osman TE, Herold R et al. Role of modeling and simulation in pediatric investigation plans. Paediatr. Anaesth. 21(3), 214-221 (2011).

37 Limdi NA, Wadelius M, Cavallari L et al. Warfarin pharmacogenetics: a single VKORC1 polymorphism is predictive of dose across 3 racial groups. Blood 115(18), 3827-3834 (2010).

38 Takahashi H. Developmental changes in pharmacokinetics and pharmacodynamics of warfarin enantiomers in Japanese children. Clin. Pharmacol. Ther. 68(5), 541-555 (2000).
39 Anderson BJ, Holford NHG. Mechanismbased concepts of size and maturity in pharmacokinetics. Annu. Rev. Pharmacol. Toxicol. 48(1), 303-332 (2008).

40 Pirmohamed M, Burnside G, Eriksson N et al. A randomized trial of genotype-guided dosing of warfarin. N. Engl. J. Med.369(24), 2294-2303 (2013).

41 Kimmel SE, French B, Kasner SE et al. A pharmacogenetic versus a clinical algorithm for warfarin dosing. N. Engl. J. Med. 369 (24), 2283-2293 (2013).

42 van Spall HGC, Wallentin L, Yusuf S et al. Variation in warfarin dose adjustment practice is responsible for differences in the quality of anticoagulation control between centers and countries: an analysis of patients receiving warfarin in the randomized evaluation of long-term anticoagulation therapy (RE-LY) Trial. Circulation 126(19), 2309-2316 (2012).

43 Monagle P, Newall F, Campbell J. Anticoagulation in neonates and children: pitfalls and dilemmas. Blood Reviews 24(4-5), 151-162 (2010).

44 Streif W, Andrew M, Marzinotto V et al. Analysis of warfarin therapy in pediatric patients: a prospective cohort study of 319 patients. Blood 94(9), 3007-3014 (1999).

45 Leeder JS, Kearns GL. Interpreting pharmacogenetic data in the developing neonate: the challenge of hitting a moving target. Clin. Pharmacol. Ther. 94, 434-436 (2012).
- Excellent review on the relative roles of pharmacogenetics and ontogeny when interpreting pharmacogenetic data in neonates.

\section{Websites}

101 US FDA approved Coumadin label. www.accessdata.fda.gov/drugsatfda_docs/ label/2010/009218s108lbl.pdf (Accessed 1 October 2013).

102 Lee JY, Madabushi R, Lesko LJ et al. Leveraging prior quantitative knowledge demonstrates the importance of genotypebased dosing of warfarin. American Conference on Pharmacometrics (2009). http://tucson2008.go-acop.org/pdfs/ Abstract_JYLee.pdf (Accessed 1 October 2013).

103 Karlsson KE. Benefits of Pharmacometric Model-Based Design and Analysis of Clinical Trials. Digital Comprehensive Summaries of Uppsala Dissertations from the Faculty of Pharmacy 133 (2010). http://uu.diva-portal.org/smash/get/ diva2:360473/FULLTEXT01.pdf (Accessed 1 October 2013).

104 Hamberg AK. Pharmacometric Models for Individualisation of Warfarin in Adults and Children. Digital Comprehensive Summaries of Uppsala Dissertations from the Faculty of Medicine 897 (2013).

http://uu.diva-portal.org/smash/get/ diva2:615277/FULLTEXT03.pdf (Accessed 1 October 2013). 